\title{
Continuous-Time Insider Trading with Risk-Neutral Insider under Imperfect Observation
}

\author{
Yonghui Zhou $\mathbb{D}^{1}{ }^{1}$ Guanglong Zhuang, ${ }^{1}$ and Kai Xiao ${ }^{2}$ \\ ${ }^{1}$ School of Big Data and Computer Science, Guizhou Normal University, Guiyang 550001, China \\ ${ }^{2}$ School of Mathematical Sciences, Guizhou Normal University, Guiyang 550001, China \\ Correspondence should be addressed to Yonghui Zhou; yonghuizhou@gznu.edu.cn
}

Received 15 July 2021; Revised 14 October 2021; Accepted 21 October 2021; Published 12 November 2021

Academic Editor: Faheem Aslam

Copyright $(92021$ Yonghui Zhou et al. This is an open access article distributed under the Creative Commons Attribution License, which permits unrestricted use, distribution, and reproduction in any medium, provided the original work is properly cited.

A model of insider trading in continuous time in which a risk-neutral insider possesses long-lived imperfect information on a risk asset is studied. By conditional expectation theory and filtering theory, we turn it into a model with insider knowing complete information about the asset with a revised risky value and deduce its linear Bayesian equilibrium consisting of optimal insider trading strategy and semistrong pricing rule. It shows that, in the equilibrium, as the degree of insider observing the signal of the risky asset value is more and more accurate, market depth, trading intensity, and residual information are all decreasing and the total expectation profit of the insider is increasing and that the information about the asset value incorporated into the equilibrium price, which has nothing to do with the volatility of noise trades, is increasing as time goes by, but not all information of asset value is incorporated into the price in the final disclosed time due to the incompleteness of insider's observation, though the market depth is still a time-independent constant. Some simulations are illustrated to show these features. However, it is an open question of how to make maximal profit if the insider is risk-averse.

\section{Introduction}

Kyle [1] first proposed a mathematical continuous auction model of insider trading and proved that as time interval goes to zero, the equilibrium of sequential trading consisting of optimal insider trading strategies and semistrong pricing rules converges to a continuous-time version in which the market depth is a time-independent constant and all private information about the asset value is incorporated into the market price. Later, Back [2] extended Kyle's continuoustime version to a general framework and gave a characterization of the insider's optima by applying dynamic programming method. And then, Cho [3] obtained a no expected trade theorem regardless of how much the insider is sensitive to the risk even for a larger class of price processes. Thanks to maximum principle, Aase et al. [4] solved directly the optimal problem of Kyle-Back's continuoustime insider trading. At the same time, Biagini et al. [5] studied the insider trading model with memory. Zhou [6] also obtained a unique linear equilibrium of continuous- time insider trading with market makers observing some partial information on a risky asset. Later, Ma et al. [7] studied the existence and uniqueness of $Q_{0}$-weak solution under reference probability measure for a more general model of continuous insider trading.

Note that the abovementioned work including other work in [8-16] on continuous-time insider trading focuses on the case that an insider knows complete information about the underlying risky asset. To our best knowledge, there are few research studies on continuous-time insider trading with insider possessing imperfect information on the risky asset, though there is much literature on discrete-time insider trading with partial information, see [17-22].

In this paper, we will study a model of continuous-time insider trading, in which a risk-neutral insider possesses imperfect information on a static risk asset traded continuously in a time interval, and investigate the impact of the accurate degree of information observed by the insider on the market equilibrium. Our main result is that it is the first time to study continuous-time insider trading with an 
insider knowing incomplete privation information on the risky asset and find that the more information the insider possesses, the more advantage the insider will take, but the information about the asset value cannot be incorporated into the market price all in the final disclosed time since the information about the asset value is not perfectly observed by the insider, while market depth, trading intensity, and residual information are all decreasing. These results are in accord with our economic intuition, which are similar to those in discrete-time insider trading [17-19]. Clearly, our model can be extended to the one with dynamic volatility of noise trades with similar results, which is indeed equivalent to some classical models of continuous-time insider trading in $[2,4,6,14,23]$. Note that, for a risk-averse insider, as in $[3,24-26]$, how to make use of imperfect information about a risky asset to maximize profit is an open question.

The rest of the paper is organized as follows. In Section 2, we propose a model of continuous-time insider trading with a risk-neutral insider under imperfect observation. In Section 3, main results about the model with its linear Bayesian equilibrium are stated. Section 4 gives some simulations. Conclusions are drawn in Section 5.

\section{The Model}

In the following, all random variables or processes are defined on a filtered probability space $\left(\Omega, \mathscr{F},\left\{\mathscr{F}_{t}\right\}, P\right)$ satisfying the usual hypothesis $[27,28]$.

As Back said in [2], in contrast to discrete-time auctions, there are three different aspects in continuous-time insider trading that we should pay attention to: (i) the insider can infer the flow of noise trades by monitoring prices continuously through without directly observing them and then can know the current price at any time; (ii) there is no advantage to observing noise trades directly for the insider; and (iii) any trading strategy of the insider that includes discrete orders or has a nonzero local martingale part does not imply that the real value is public at the end. Therefore, the above three reasonable intuitions will also be integrated into our model framework.

The model of insider trading here is a continuous version of the model with a risky neutral insider as in [22]. Similar to $[1,2,6,22]$, we assumed that there is a risk asset traded in a finite time interval $[0, T]$, whose value $v$ is normally distributed $N\left(0, \sigma_{v}^{2}\right)$ and disclosed at the final time $T$. Also, there are three types of agents:

(1) Noise traders, or liquidities, who have no information about the risk asset value $v$ and randomly submit a total order $z_{t}$ at time, which is assumed as in [3] that $z_{t}=\sigma_{z} B_{t}$, where $z_{0}=0, \sigma_{z}>0$, and $B_{t}$ is a standard Brownian motion independent of $v$

(2) A risk-neutral insider, who at time $t$ knows both the current price $p_{t}$ of the asset and incomplete information on the asset value as $v+\varepsilon$, where the noise $\varepsilon \sim N\left(0, \sigma_{\varepsilon}^{2}\right)$ is independent of both asset value $v$ and liquidity trades $z$, and according to the information denoted by $\mathscr{F}_{t}^{I}$ submits an order $x_{t}$ to maximize her/ his profit, where $x_{0}=0$
(3) Marked makers, who at time $t$ observe the total traded volume $y_{t}=x_{t}+z_{t}$ (but cannot discriminate $x_{t}$ and $z_{t}$, respectively) and according to the observation information denoted by $\mathscr{F}_{t}^{M}$ set market price $p_{t}$ for the risk asset value in a semistrong efficient way [1]

So, the insider is self-financing [29] and adopts a trading strategy $x$ with differentiable paths. The collection of insider's trading volume functions $x$ and the collection of market makers' effective pricing functions $p$ are denoted by $X$ and $P$, respectively. Clearly, given a pair $(x, p) \in(X, P)$, the insider's profit is $\int_{0}^{T}\left(v-p_{t}\right) \mathrm{d} x_{t}$ [2]. Then, a market equilibrium is a pair of stochastic processes $(x, p) \in(X, P)$.

Definition 1. A linear equilibrium is defined as a pair $(x, p) \in(X, P)$ such that the following conditions hold:

(i) Maximization of profit: given the price rule $p$, the strategy $x$ maximizes

$$
E\left[\int_{0}^{T}\left(v-p_{t}\right) \mathrm{d} x_{t} \mid \mathscr{F}_{t}^{I}\right] .
$$

(ii) Market efficiency: given the strategy $x$, for any time $t$, the price

$$
p_{t}=E\left[v \mid \mathscr{F}_{t}^{M}\right]
$$

\section{Main Results}

In the following, we would like to turn the above model into an equivalent market with insider knowing complete information about the asset with a revised risky value and then find some kind of linear equilibrium. To do this, we need some preparations.

In the model, for any time $t$, the insider's and market makers' information can be written, respectively, as

$$
\begin{aligned}
\mathscr{F}_{t}^{I} & =\mathscr{F}^{v+\varepsilon} \vee \mathscr{F}_{t}^{p}, \\
\mathscr{F}_{t}^{M} & =\mathscr{F}_{t}^{y} .
\end{aligned}
$$

Lemma 1. Equivalent price.

$$
E\left[v \mid \mathscr{F}_{t}^{M}\right]=E\left[\frac{\sigma_{v}^{2}}{\sigma_{v}^{2}+\sigma_{\varepsilon}^{2}}(v+\varepsilon) \mid \mathscr{F}_{t}^{M}\right]
$$

Proof. Since there are only two noisy signals in the market, one is the insider's observation $v+\varepsilon$ and the other is the Brownian motion $B$ with respect to the noise order $z$, then for market makers, there must be

$$
\mathscr{F}_{t}^{M}=\mathscr{F}_{t}^{y} \subset \mathscr{F}_{t}^{B} \vee \mathscr{F}^{v+\varepsilon} .
$$

Then, 


$$
\begin{aligned}
E\left[v \mid \mathscr{F}_{t}^{M}\right] & =E\left[v \mid \mathscr{F}_{t}^{y}\right] \\
& =E\left[E\left[v \mid \mathscr{F}_{t}^{B} \vee \mathscr{F}^{v+\varepsilon}\right] \mid \mathscr{F}_{t}^{y}\right] \\
& =E\left[E[v \mid v+\varepsilon] \mid \mathscr{F}_{t}^{M}\right] \\
& =E\left[\frac{\sigma_{v}^{2}}{\sigma_{v}^{2}+\sigma_{\varepsilon}^{2}}(v+\varepsilon) \mid \mathscr{F}_{t}^{M}\right],
\end{aligned}
$$

where the second equality follows from the tower law of conditional expectation $[30,31]$, the third follows from the independence between the asset value $v$ and the Brownian motion $B$, and the last equality follows from the filtering of normal distribution [32].

Lemma 2. Equivalent profit.

$$
E\left[\int_{0}^{T}\left(v-p_{t}\right) \mathrm{d} x_{t} \mid \mathscr{F}_{t}^{I}\right]=E\left[\int_{0}^{T}\left(\frac{\sigma_{v}^{2}}{\sigma_{v}^{2}+\sigma_{\varepsilon}^{2}}(v+\varepsilon)-p_{t}\right) \mathrm{d} x_{t} \mid \mathscr{F}_{t}^{I}\right] .
$$

Proof. The proof is similar to that of Lemma 1.

By integration by parts,

$$
\begin{aligned}
& E\left[\int_{0}^{T}\left(v-p_{t}\right) \mathrm{d} x_{t} \mid \mathscr{F}_{t}^{I}\right] \\
& =E\left[\left(v-p_{T}\right) x_{T}+\int_{0}^{T} x_{t} \mathrm{~d} p_{t} \mid \mathscr{F}_{t}^{I}\right] \\
& =E\left[\left(v-p_{T}\right) x_{T} \mid \mathscr{F}_{t}^{I}\right]+E\left[\int_{0}^{T} x_{t} \mathrm{~d} p_{t} \mid \mathscr{F}_{t}^{I}\right] .
\end{aligned}
$$

Since for any time $t$

$$
\mathscr{F}_{t}^{I} \subset F_{T}^{B} \vee \mathscr{F}^{v+\varepsilon},
$$

both $x_{T}$ and $p_{T}$ are measurable with respect to $F_{T}^{B} \vee \mathscr{F}^{v+\varepsilon}$ and $v$ is independent of the Brownian motion $B$, then from the expression of (8) and by the tower law of conditional expectation and filtering of normal distribution,

$$
\begin{aligned}
E\left[\left(v-p_{T}\right) x_{T} \mid \mathscr{F}_{t}^{I}\right] & =E\left[E\left[\left(v-p_{T}\right) x_{T} \mid F_{T}^{B} \vee \mathscr{F}^{v+\varepsilon}\right] \mid \mathscr{F}_{t}^{I}\right] \\
& =E\left[\left(\left[v \mid F_{T}^{B} \vee \mathscr{F}^{v+\varepsilon}\right]-p_{T}\right) x_{T} \mid \mathscr{F}_{t}^{I}\right] \\
& =E\left[\left(E[v \mid v+\varepsilon]-p_{T}\right) x_{T} \mid \mathscr{F}_{t}^{I}\right] \\
& =E\left[\left(\frac{\sigma_{v}^{2}}{\sigma_{v}^{2}+\sigma_{\varepsilon}^{2}}(v+\varepsilon)-p_{T}\right) x_{T} \mid \mathscr{F}_{t}^{I}\right] .
\end{aligned}
$$

Therefore, combining this with (8), we have

$$
\begin{aligned}
E\left[\int_{0}^{T}\left(v-p_{t}\right) \mathrm{d} x_{t} \mid \mathscr{F}_{t}^{I}\right] & =E\left[\left(\frac{\sigma_{v}^{2}}{\sigma_{v}^{2}+\sigma_{\varepsilon}^{2}}(v+\varepsilon)-p_{T}\right) x_{T} \mid \mathscr{F}_{t}^{I}\right]+E\left[\int_{0}^{T} x_{t} \mathrm{~d} p_{t} \mid \mathscr{F}_{t}^{I}\right] \\
& =E\left[\left(\frac{\sigma_{v}^{2}}{\sigma_{v}^{2}+\sigma_{\varepsilon}^{2}}(v+\varepsilon)-p_{T}\right) x_{T}+\int_{0}^{T} x_{t} \mathrm{~d} p_{t} \mid \mathscr{F}_{t}^{I}\right] \\
& =E\left[\int_{0}^{T}\left(\frac{\sigma_{v}^{2}}{\sigma_{v}^{2}+\sigma_{\varepsilon}^{2}}(v+\varepsilon)-p_{t}\right) \mathrm{d} x_{t} \mid \mathscr{F}_{t}^{I}\right],
\end{aligned}
$$

where the last equality follows again by integration by parts, and the proof is completed.

By Lemmas 1 and 2, our model with insider knowing partial information $v+\varepsilon$ becomes a continuous model with insider knowing complete information as $\left(\sigma_{v}^{2} /\left(\sigma_{v}^{2}+\sigma_{\varepsilon}^{2}\right)\right)(v+\varepsilon)$. So, one can apply dynamic programming $[2,3]$ or maximum principle $[4,6,23]$ to find some kind of linear equilibrium. Therefore, we here only consider such linear strategies $(x, p)$ with $(\beta, \lambda)$ satisfying

$$
\mathrm{d} x_{t}=\beta(t)\left((v+\varepsilon)-p_{t}\right) \mathrm{d} t, \quad \mathrm{~d} p_{t}=\lambda_{t} \mathrm{~d} y_{t},
$$

where $\beta$ and $\lambda$ are deterministic function on $[0, T]$, with its proof omitted.

Theorem 1 (linear equilibrium). There is a unique linear equilibrium $(\beta, \lambda)$ with their dynamics by

$$
x_{t}=\int_{0}^{t} \beta(t)\left(\frac{\sigma_{v}^{2}}{\sigma_{v}^{2}+\sigma_{\varepsilon}^{2}}(v+\varepsilon)-p_{t}\right) \mathrm{d} t, \quad p_{t}=\lambda(t) y_{t},
$$

where 


$$
\lambda(t) \equiv \frac{\sigma_{v}^{2}}{\sigma_{z} \sqrt{T\left(\sigma_{v}^{2}+\sigma_{\varepsilon}^{2}\right)}} .
$$

At the equilibrium, the insider's ex-post profit from time $t$ to the end time $T$

$$
\begin{aligned}
& E\left[\int_{t}^{T}\left(v-p_{t}\right) d x_{t} \mid \mathscr{F}_{t}^{I}\right] \\
& =\frac{\sigma_{z} \sqrt{\left(\sigma_{v}^{2}+\sigma_{\varepsilon}^{2}\right) T}\left(\left(\sigma_{v}^{2} /\left(\sigma_{v}^{2}+\sigma_{\varepsilon}^{2}\right)\right)(v+\varepsilon)-p_{t}\right)^{2}}{2 \sigma_{v}^{2}}+\frac{\sigma_{z} \sigma_{v}^{2}(T-t)}{2 \sqrt{\left(\sigma_{v}^{2}+\sigma_{\varepsilon}^{2}\right) T}},
\end{aligned}
$$

and the insider's ex-ante profit from the beginning to the end of the insider

$$
E(P)=E\left[\int_{0}^{T}\left(v-p_{t}\right) \mathrm{d} x_{t}\right]=\frac{\sigma_{z} \sigma_{v}^{2} \sqrt{T}}{\sqrt{\sigma_{z}^{2}+\sigma_{\varepsilon}^{2}}}
$$

Remark 1. By Theorem 1,

(i) $(\mathrm{d} \beta(t) / \mathrm{d} t)>0,(\mathrm{~d}(1 / \lambda) / \mathrm{d} t)=0$

(ii) $\left(\mathrm{d} \beta(t) / \mathrm{d} \sigma_{\varepsilon^{2}}\right)>0,\left(\mathrm{~d}(1 / \lambda(t)) / \mathrm{d} \sigma_{\varepsilon^{2}}\right)<0,\left(\mathrm{~d} E\left[\int_{0}^{T}(v-\right.\right.$ $\left.\left.\left.p_{t}\right) \mathrm{d} x_{t}\right] / \mathrm{d} \sigma_{\varepsilon^{2}}\right)<0$

(iii) $\lim _{\sigma_{\varepsilon}^{2} \longrightarrow \infty} \beta(t)=\infty, \lim _{\sigma_{\varepsilon}^{2}} \longrightarrow \infty(1 / \lambda(t))=\infty$, $\lim _{\sigma_{\varepsilon}^{2} \rightarrow \infty} E\left[\int_{0}^{T}\left(v-p_{t}\right) \mathrm{d} x_{t}\right]=0$
That is to say, (i) trading intensity $\beta$ increases with time $t$ while $(1 / \lambda)$, called market depth [1], is time-independent; (ii) the more accurate the information on the risky asset observed by the insider, the less trading intensity $\beta$ is while the deeper market depth $(1 / \lambda)$ is such that the insider can make higher positive profit; (iii) furthermore, the insider can earn maximum profit when observing the asset value $v\left(\sigma_{\varepsilon}=0\right)$, and with $\sigma_{\varepsilon}^{2}$ increasing, both trading intensity and market depth increase even to $\infty$; if $\sigma_{\varepsilon} \longrightarrow \infty$, however, insiders' profit is decreasing even to 0 ; in this case, the insider basically has no private information. In other words, the insider has become a noise trader. This is completely consistent with our intuition.

Clearly, our results when $\sigma_{\varepsilon}=0$ and $T=1$ are the same as those in models with insider knowing complete information about the asset value $[1,3,6,7]$.

Proposition 1 (residual information). Let $(x, p)$ be the linear equilibrium in Theorem 1, and denote $\Sigma_{t}=E\left[\left(v-p_{t}\right)^{2}\right]$. Then,

$$
\Sigma_{t}=\sigma_{v}^{2}-\frac{\sigma_{v}^{4}}{\sigma_{v}^{2}+\sigma_{\varepsilon}^{2}} \frac{t}{T}
$$

Proof. Let $(x, p)$ be the linear equilibrium in Theorem 1. To compute the variance $\Sigma_{t}=E\left[\left(v-p_{t}\right)^{2}\right]$, we need the help of Kalman-Bucy filtering theory.

In the model, for market makers, there is a signal-observation system:

signals: $\mathrm{d} v_{t}=0, v_{0}=v ; \mathrm{d} \varepsilon_{t}=0, \varepsilon_{0}=\varepsilon$,

$$
\text { observation: } \mathrm{d} y_{t}=\mathrm{d} x_{t}+\mathrm{d} z_{t}=\beta(t)\left(\frac{\sigma_{v}^{2}}{\sigma_{v}^{2}+\sigma_{\varepsilon}^{2}}(v+\varepsilon)-p_{t}\right) \mathrm{d} t+\sigma_{z} \mathrm{~d} B_{t} \text {. }
$$

Denote the optimal filter of the signal $\theta_{t}$ by $\widehat{\theta}_{t}=\left(p_{t}, q_{t}\right)^{*}$ with $p_{t}=E\left[v \mid \mathscr{F}_{t}^{y}\right]$ and $q_{t}=E\left[\mathcal{E} \mid \mathscr{F}_{t}^{y}\right]$ and the covariance matrix $S_{t}=E\left[\left(\theta_{t}-\widehat{\theta}\right)\left(\theta_{t}-\widehat{\theta}\right)^{*}\right]=\left(\begin{array}{cc}S_{1 t} & S_{12 t} \\ S_{12 t} & S_{2 t}\end{array}\right)$ at time $t$. Then, $p_{0}=0, q_{0}=0$ and $S_{0}=\left(\begin{array}{cc}\sigma_{v}^{2} & 0 \\ 0 & \sigma_{\varepsilon}^{2}\end{array}\right)$.

Also, denote $G_{t}=\beta(t)\left(\sigma_{v}^{2} /\left(\sigma_{v}^{2}+\sigma_{\varepsilon}^{2}\right)\right)(1,1)$. Then, by Theorem 12.7 in [32] or Theorem 6.3.1 in [33], it shows that the dynamics of $\widehat{\theta}_{t}$ and $S_{t}$ are, respectively, as

$$
\left\{\begin{array}{l}
\mathrm{d} \widehat{\theta}_{t}=\frac{1}{\sigma_{z}^{2}} S_{t} G^{*} \mathrm{~d} y_{t}, \\
\frac{\mathrm{d} S_{t}}{\mathrm{~d} t}=-\frac{1}{\sigma_{z}^{2}} S_{t} G_{t}^{*} G_{t} S_{t} .
\end{array}\right.
$$

Note that, in (19), the second vector-equation can be written in the form of components as follows:

$$
\left\{\begin{array}{l}
\frac{\mathrm{d} S_{1 t}}{\mathrm{~d} t}=-\widetilde{\beta}_{t}^{2}\left(S_{1 t}+S_{12 t}\right)^{2} \\
\frac{\mathrm{d} S_{12 t}}{\mathrm{~d} t}=-\widetilde{\beta}_{t}^{2}\left(S_{1 t}+S_{12 t}\right)\left(S_{2 t}+S_{12 t}\right) \\
\frac{\mathrm{d} S_{2 t}}{\mathrm{~d} t}=-\widetilde{\beta}_{t}^{2}\left(S_{2 t}+S_{12 t}\right)^{2}
\end{array}\right.
$$

where $\widetilde{\beta}_{t}=\left(\beta(t) \sigma_{v}^{2} / \sigma_{z}\left(\sigma_{v}^{2}+\sigma_{\varepsilon}^{2}\right)\right)$. To solve the equations, we introduce two notations:

$$
\left\{\begin{array}{l}
r_{1 t}=S_{1 t}+S_{12 t} \\
r_{2 t}=S_{2 t}+S_{12 t}
\end{array}\right.
$$

Then, from (20) and (21), we can obtain the following two equations: 


$$
\left\{\begin{array}{l}
\frac{\mathrm{d}\left(r_{1 t}+r_{2 t}\right)}{\mathrm{d} t}=-\widetilde{\beta}_{t}^{2}\left(r_{1 t}+r_{2 t}\right)^{2} \\
\frac{\mathrm{d}\left(r_{1 t}-r_{2 t}\right)}{\mathrm{d} t}=-\widetilde{\beta}_{t}^{2}\left(r_{1 t}-r_{2 t}\right)\left(r_{1 t}+r_{2 t}\right)
\end{array}\right.
$$

where $\quad r_{10}+r_{20}=S_{0 t}+2 S_{120}+S_{20}=\sigma_{v}^{2}+\sigma_{\varepsilon}^{2}$ and $r_{10}-r_{20}=\sigma_{v}^{2}-\sigma_{\varepsilon}^{2}$. Also, it is easy to show that

$$
\begin{aligned}
& r_{1 t}+r_{2 t}=\frac{1}{\left(1 /\left(\sigma_{v}^{2}+\sigma_{\varepsilon}^{2}\right)\right)+\int_{0}^{t} \widetilde{\beta}_{t}^{2} \mathrm{~d} t}, \\
& r_{1 t}-r_{2 t}=\frac{\sigma_{v}^{2}-\sigma_{\varepsilon}^{2}}{\sigma_{v}^{2}+\sigma_{\varepsilon}^{2}} \frac{1}{\left(1 /\left(\sigma_{v}^{2}+\sigma_{\varepsilon}^{2}\right)\right)+\int_{0}^{t} \widetilde{\beta}_{t}^{2} \mathrm{~d} t} .
\end{aligned}
$$

Therefore, we have

$$
\begin{aligned}
& r_{1 t}=\frac{\sigma_{v}^{2}}{\sigma_{v}^{2}+\sigma_{\varepsilon}^{2}} \frac{1}{\left(1 /\left(\sigma_{v}^{2}+\sigma_{\varepsilon}^{2}\right)\right)+\int_{0}^{t}\left(\beta_{t}^{2} / \sigma_{t}^{2}\right) \mathrm{d} t}, \\
& r_{2 t}=\frac{\sigma_{\varepsilon}^{2}}{\sigma_{v}^{2}+\sigma_{\varepsilon}^{2}} \frac{1}{\left(1 /\left(\sigma_{v}^{2}+\sigma_{\varepsilon}^{2}\right)\right)+\int_{0}^{t}\left(\beta_{t}^{2} / \sigma_{t}^{2}\right) \mathrm{d} t} .
\end{aligned}
$$

Finally, the explicit forms of $S_{1 t}, S_{12 t}$, and $S_{2 t}$ can be written easily as follows:

$$
\begin{aligned}
& S_{1 t}=\frac{\sigma_{v}^{4}}{\left(\sigma_{v}^{2}+\sigma_{\varepsilon}^{2}\right)^{2}} \frac{1}{\left(1 /\left(\sigma_{v}^{2}+\sigma_{\varepsilon}^{2}\right)\right)+\int_{0}^{t} \tilde{\beta}_{t}^{2} \mathrm{~d} t}+\frac{\sigma_{v}^{2} \sigma_{\varepsilon}^{2}}{\sigma_{v}^{2}+\sigma_{\varepsilon}^{2}}, \\
& S_{12 t}=\frac{\sigma_{v}^{2} \sigma_{\varepsilon}^{2}}{\left(\sigma_{v}^{2}+\sigma_{\varepsilon}^{2}\right)^{2}} \frac{1}{\left(1 /\left(\sigma_{v}^{2}+\sigma_{\varepsilon}^{2}\right)\right)+\int_{0}^{t} \tilde{\beta}_{t}^{2} \mathrm{~d} t}-\frac{\sigma_{v}^{2} \sigma_{\varepsilon}^{2}}{\sigma_{v}^{2}+\sigma_{\varepsilon}^{2}}, \\
& S_{2 t}=\frac{\sigma_{\varepsilon}^{4}}{\left(\sigma_{v}^{2}+\sigma_{\varepsilon}^{2}\right)^{2}} \frac{1}{\left(1 /\left(\sigma_{v}^{2}+\sigma_{\varepsilon}^{2}\right)\right)+\int_{0}^{t} \tilde{\beta}_{t}^{2} \mathrm{~d} t}+\frac{\sigma_{v}^{2} \sigma_{\varepsilon}^{2}}{\sigma_{v}^{2}+\sigma_{\varepsilon}^{2}} .
\end{aligned}
$$

So, taking the value of $\widetilde{\beta}_{t}$ above, we obtain

$$
\Sigma_{t}=E\left[\left(v-p_{t}\right)^{2}\right]=S_{1 t}=\sigma_{v}^{2}-\frac{\sigma_{v}^{4}}{\sigma_{v}^{2}+\sigma_{\varepsilon}^{2}} \frac{t}{T} .
$$

Remark 2. Note that the variance $\Sigma_{t}$ describes the informativeness of the price [1] at time $t$ and then $I(t)=\sigma_{v}^{2}-\Sigma_{t}$ measures how much information has been incorporated into the equilibrium price [18]. Here, in our model at time $t$,

$$
I(t)=\frac{\sigma_{v}^{4}}{\sigma_{v}^{2}+\sigma_{\varepsilon}^{2}} \frac{t}{T}
$$

and then

$$
\begin{aligned}
& \frac{\mathrm{d} I(t)}{\mathrm{d} t}>0, \\
& I(T)=\frac{\sigma_{v}^{4}}{\sigma_{v}^{2}+\sigma_{\varepsilon}^{2}}<\sigma_{v}^{2},
\end{aligned}
$$

which means that the information about the asset value incorporated into the equilibrium price is increasing as time goes by but not all information about the asset value is incorporated into the price at the end time when the asset value becomes public, except complete information observed by the insider $\left(I(T)=0\right.$ if $\sigma_{\varepsilon}$ approaching $\left.\infty\right)$; this is because of the insider's incomplete observation on the asset value.

Note that, by expression (26), for any trade time $t$, the residual information $\Sigma_{t}$ is only related to the variance $\sigma_{v}^{2}$ of the risky asset value $v$ and the variance $\sigma_{\varepsilon}^{2}$ of the insider's observation noise $\varepsilon$, but not to the volatility $\sigma_{z}$ of noise trading volume $z$. In fact,

$$
\begin{gathered}
\frac{\mathrm{d} \Sigma_{t}}{\mathrm{~d} t}<0, \\
\frac{\mathrm{d} \Sigma_{t}}{\mathrm{~d} \sigma_{\mathcal{\varepsilon}^{2}}}>0, \\
\lim _{t \longrightarrow T} \Sigma_{t}=\frac{\sigma_{v}^{2} \sigma_{\varepsilon}^{2}}{\sigma_{v}^{2}+\sigma_{\varepsilon}^{2}}, \\
\lim _{\sigma_{\varepsilon}^{2} \longrightarrow \infty} \Sigma_{t}=\sigma_{v^{2}}^{2}
\end{gathered}
$$

Remark 3. From equation (17), we have

$$
\Sigma_{T}=\frac{\sigma_{v}^{2} \sigma_{\varepsilon}^{2}}{\sigma_{v}^{2}+\sigma_{\varepsilon}^{2}} .
$$

Then, $\Sigma_{T} \neq 0$ if and only if $\sigma_{\varepsilon} \neq 0\left(\sigma_{v} \neq 0\right)$. It shows that, as long as the insider's information is imperfect, the residual information will never be released completely.

\section{Simulations}

To see some propositions of parameters, including trading intensity, market depth, residual information, and insider's profit, varying with volatility of noise observed by the insider or trading time at the equilibrium in our model more clearly, we give some simulations according to our Theorem 1 or Proposition 1.

In the following, consider the continuous trading time $t \in[0,1]$ (the trading final time $T=1$ ), the variance of the risky asset value $\sigma_{v}^{2}=1$, and the volatility of the noisy trading order $\sigma_{z}^{2}=1$. Then, at equilibrium, trading intensity, market depth, residual information, and insider's profit can be written using Theorem 1 or Proposition 1 as follows:

$$
\begin{aligned}
\beta_{t} & =\frac{\sqrt{1+\sigma_{\varepsilon}^{2}}}{1-t}, \\
\frac{1}{\lambda_{t}} & =\sqrt{1+\sigma_{\varepsilon}^{2}}, \\
\Sigma_{t} & =1-\frac{t}{1+\sigma_{\varepsilon}^{2}}, \\
E(P) & =\frac{1}{\sqrt{1+\sigma_{\varepsilon}^{2}}} .
\end{aligned}
$$




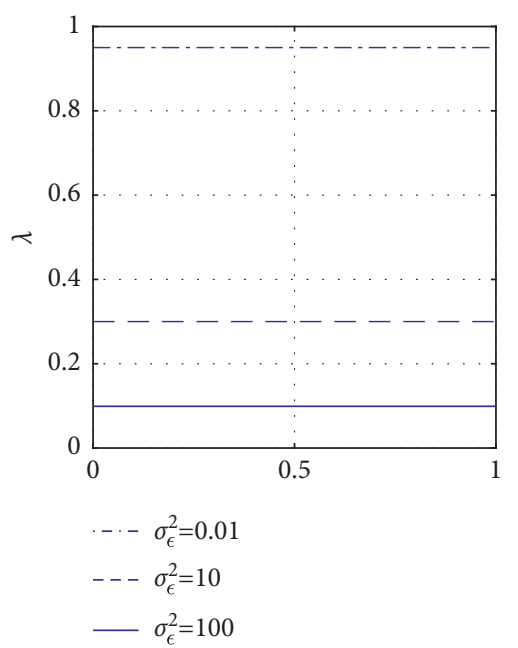

(a)

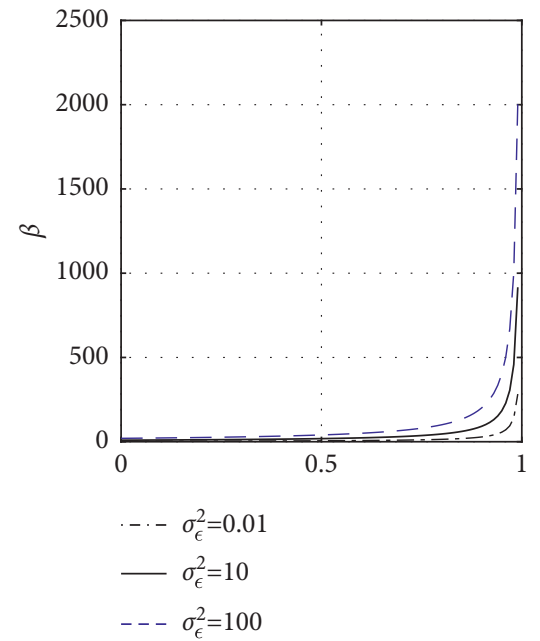

(b)

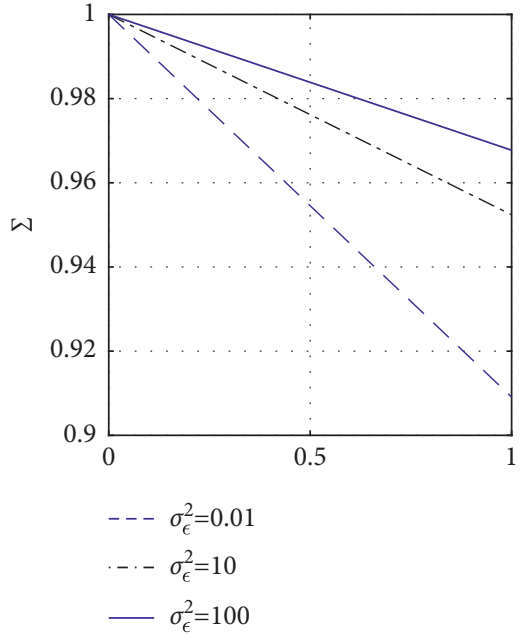

(c)

FIGURE 1: Market liquidity $\lambda$, trading intensity $\beta$, and residual information $\Sigma_{t}$ varying with time $t$ and different degrees of volatility $\sigma_{\varepsilon}^{2}$ of noise in risk asset value. (a) $\lambda$ varying with $t$. (b) $\beta$ varying with $t$. (c) $\Sigma$ varying with $t$.

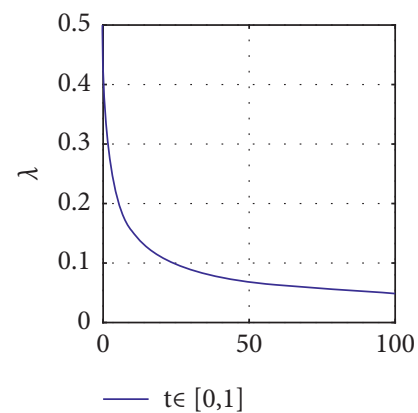

(a)

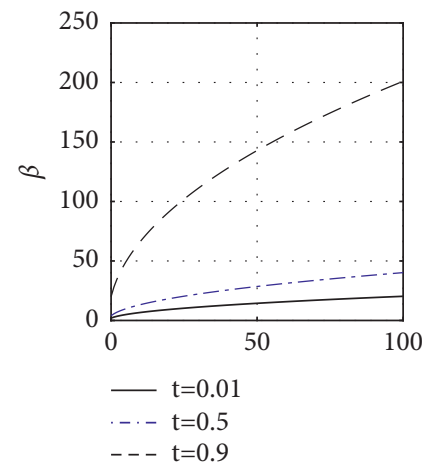

(b)

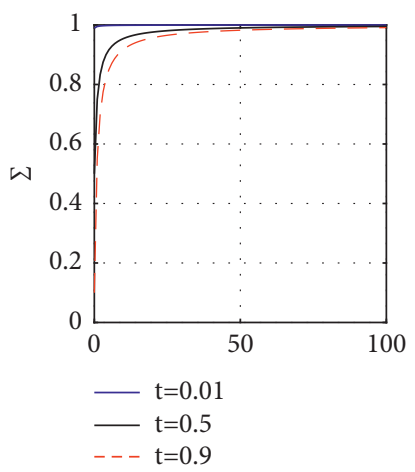

(c)

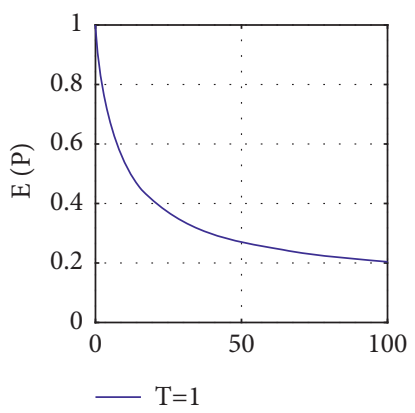

(d)

FIGURE 2: Market liquidity $\lambda$, trading intensity $\beta$, residual information $\Sigma$, and insider's total expectation profit $E(P)$ varying with volatility $\sigma_{\varepsilon}^{2}$ of noise in risk asset value at different time $t$. (a) $\lambda$ varying with $\sigma_{\varepsilon}^{2}$. (b) $\beta$ varying with $\sigma_{\varepsilon}^{2}$. (c) $\Sigma$ varying with $\sigma_{\varepsilon}^{2}$. (d) $E(P)$ varying with $\sigma_{\varepsilon}^{2}$.

Figure 1 shows that, at the equilibrium for any information $v+\varepsilon$ with noise $\varepsilon \sim N\left(0, \sigma_{\varepsilon}^{2}\right)$ received by the insider, it is easy to see that w.r.t time $t$, the market liquidity is always constant (Figure $1(\mathrm{a})$ ); the transaction intensity $\beta$ is concave and increasing to $\infty$ when the time approaching the final time $T=1$ (Figure 1(b)); and the residual information is decreasing linearly but $\Sigma_{1}>0$ since the insider does not observe the whole information on the risky asset (Figure 1(c)).

Figure 2 shows that, for any time $t \in[0,1]$, as volatility $\sigma_{\varepsilon}^{2}$ of the observing noise in risky asset value decreases, that is to say, the degree of insider observing the signal of the risky asset value is more and more accurate, market depth $1 / \lambda$ (Figure 2(a)), trading intensity $\beta_{t}$, and residual information $\Sigma_{t}$ are all decreasing (Figures 2(b) and 2(c)), while the total expectation profit $E(P)$ of the insider is increasing (Figure 2(d)). These results can be understood since the more accurate the information held by the insider, the deeper the market depth is (the weaker the trading intensity and the less the residual information) such that the insider makes more profit.

\section{Conclusions}

In this paper, we study an extended Kyle-Back's model in continuous-time insider trading, which allows a risk-neutral insider to possess long-lived imperfect privative information about a static risk asset traded in a finite time interval.

By applying conditional expectation theory and filtering theory, we first establish two lemmas: equivalent price (Lemma 1) and equivalent profit (Lemma 2), which can turn our model into a model with insider knowing complete information about the asset with a revised risky value. Then, by some existing results in the literature $([2,3,14,23]$ or $[4,6])$, we deduce a closed form of its linear equilibrium consisting of semistrong pricing rule and optimal insider trading strategy with insider's ex-post and ex-ante profits (Theorem 1). 
It shows that, in the equilibrium, as the degree of the signal of the risky asset value observed by the insider is more and more accurate, market depth, trading intensity, and residual information are all decreasing and the total expectation profit of the insider is increasing and that the information about the asset value incorporated into the equilibrium price, which has nothing to do with the volatility of noise trades, is increasing as time goes by but not all information of asset value is incorporated into the price in the final disclosed time due to the incompleteness of insider's observation, though the market depth is still a timeindependent constant (Remark 1). Finally, by using the vector Kalman-Bucy filtering method, we find that the information about the asset value incorporated into the equilibrium price is increasing as time goes by, but not all of its information is incorporated into the price in the final disclosed time due to the incompleteness of insider's observation (Proposition 1 and Remark 2). These results are in accord with our economic intuition, which are similar to those for discrete-time insider trading [17-19].

As pointed out in Section 2, our model of continuoustime insider trading with a risk-neutral insider, which can be extended to the one with dynamic volatility of noise trades with similar results, is indeed equivalent to some classical models of continuous-time insider trading in $[2,3,14,23]$ or $[4,6]$. However, for a risk-averse insider, how to make use of incomplete information about a risky asset to maximize profit is an open question, since our result in Lemma 2 will not be valid for risk-averse utility, which leaves to the readers.

\section{Data Availability}

No data were used to support this study.

\section{Conflicts of Interest}

The authors declare that they have no conflicts of interest.

\section{Acknowledgments}

This work was supported by NSF China (11861025) and Guizhou QKHPTRC[2018]5769.

\section{References}

[1] A. S. Kyle, "Continuous auctions and insider trading," Econometrica, vol. 53, no. 6, pp. 1315-1335, 1985.

[2] K. Back, "Insider trading in continuous time," Review of Financial Studies, vol. 5, no. 3, pp. 387-409, 1992.

[3] K.-H. Cho, "Continuous auctions and insider trading: uniqueness and risk aversion," Finance and Stochastics, vol. 7, no. 1, pp. 47-71, 2003.

[4] K. K. Aase, T. Bjuland, and B. Øksendal, "Strategic insider trading equilibrium: a filter theory approach," Afrika Matematika, vol. 23, no. 2, pp. 145-162, 2012.

[5] F. Biagini, Y. Hu, T. Meyer-Brandis, and B. Øksendal, "Insider trading equilibrium in a market with memory," Mathematics and Financial Economics, vol. 6, no. 3, pp. 229-247, 2012.
[6] Y. Zhou, "Existence of linear strategy equilibrium in insider trading with partial observations," Journal of Systems Science and Complexity, vol. 29, no. 5, pp. 1281-1292, 2016.

[7] J. Ma, R. Sun, and Y. Zhou, "Kyle--Back equilibrium models and linear conditional mean-field SDEs," SIAM Journal on Control and Optimization, vol. 56, no. 2, pp. 1154-1180, 2018.

[8] P. Collins-Dufresne and V. Fos, "Insider trading, stochastic liquidity and equilibrium prices," Econometrica, vol. 84, pp. 14451-21475, 2016.

[9] A. Kohatsu-Higa and S. Ortiz-Latorre, "Modeling of financial markets with inside information in continuous time," Stochastics and Dynamics, vol. 11, no. 2n03, pp. 415-438, 2011.

[10] R. Caldentey and E. Stacchetti, "Insider trading with a random deadline," Econometrica, vol. 1, pp. 245-283, 2010.

[11] A. Danilova, "Stock market insider trading in continuous time with imperfect dynamic information," Stochastics, vol. 82, no. 1, pp. 111-131, 2010.

[12] L. Campi, U. Çetin, and A. Danilova, "Dynamic markov bridges motivated by models of insider trading," Stochastic Processes and Their Applications, vol. 121, no. 3, pp. 534-567, 2011.

[13] L. Campi, U. Çetin, and A. Danilova, "Equilibrium model with default and dynamic insider information," Finance and Stochastics, vol. 17, no. 3, pp. 565-585, 2013.

[14] K. Back and H. Pedersen, "Long-lived information and intraday patterns," Journal of Financial Markets, vol. 1, no. 3-4, pp. 385-402, 1998.

[15] K. Xiao and Y. Zhou, "Insider trading with memory under random deadline," Journal of Mathematics, vol. 2021, Article ID 2973361, 7 pages, 2021

[16] K. Xiao and Y. Zhou, "Insider trading with a random deadline under partial observations: maximal principle method," Acta Mathematicae Applicatae Sinica, 2021.

[17] N. Jain and L. J. Mirman, "Insider trading with correlated signals," Economics Letters, vol. 65, no. 1, pp. 105-113, 1999.

[18] S. Luo, "The impact of public information on insider trading," Economics Letters, vol. 70, no. 1, pp. 59-68, 2001.

[19] D. Zhou, "Overconfidence on public information," Economics Letters, vol. 112, no. 3, pp. 239-242, 2011.

[20] H. Chen and B. Jorgensen, "Insider trading, competition, and real activities manipulation," Management Science (Online), pp. 1-15, 2021.

[21] A. Buffa, "Strategic insider trading with imperfect information: a trading volume Analysis," Rivista di Politica Economica, vol. 94, pp. 101-143, 2004.

[22] X. Ji and F. Gong, "The equilibrium and its asymptotic analysis of insider trading model under the incomplete information with a risk-seeking insider trader," Acta Mathematicae Applicatae Sinica, vol. 37, pp. 304-312, 2014.

[23] J. Yong and X. Zhou, Stochastic Controls, Springer, Berlin, Germany, 1999.

[24] S. Baruch, "Insider trading and risk aversion," Journal of Financial Markets, vol. 5, no. 4, pp. 451-464, 2002.

[25] G. Nunno, J. Corcuera, B. Øksendal, and G. Farkas, "KyleBack's model with Levy noise," Pure Mathematics, 2010.

[26] B. H. Lim, "A risk-averse insider and asset pricing in continuous time," Management Science and Financial Engineering, vol. 19, no. 1, pp. 11-16, 2013.

[27] P. Protter, Stochastic Integration and Differential Equations-A New Approach, Springer-Verlag, Berlin, Germany, 1990.

[28] P. Billingsley, Probability and Measure, John Wiley \& Sons, Hoboken, NJ, USA, 1995.

[29] T. Björk, Arbitrage Theory in Continuous Time, Oxford University, New York, NY, USA, 2009. 
[30] M. Capinski, E. Kopp, and J. Traple, Stochastic Calculus for Finance, Cambridge University Press, Cambridge, UK, 2012.

[31] E. Kopp, J. Malczak, and T. Zastawniak, Probability for Finance, Cambridge University Press, Cambridge, UK, 2014.

[32] R. Liptser and A. Shiryaev, Statistic of Random Process I. General Theory, Springer, Berlin, Germany, 2001.

[33] B. Øksendal, Stochastic Differential Equations, Springer, Berlin, Germany, 2003. 